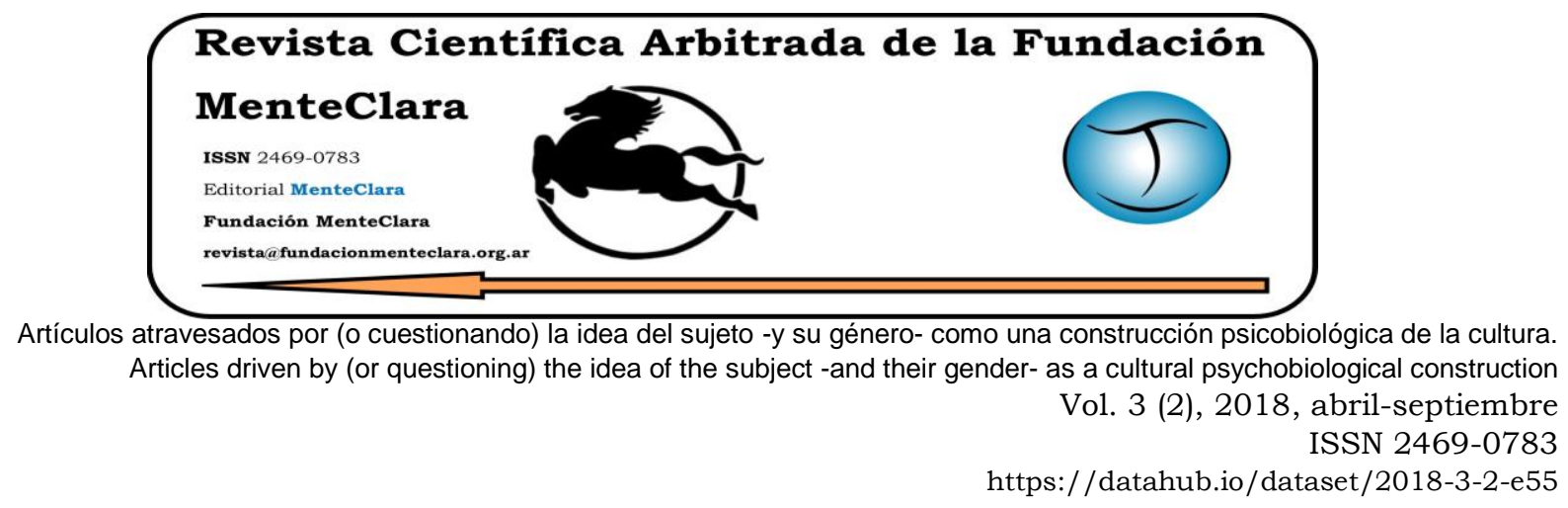

\title{
IDEOLOGÍA DEL ROL DE GÉNERO, ESPIRITUALIDAD Y RELIGIOSIDAD EN CONTEXTOS MILITARES
}

\author{
GENDER ROLE IDEOLOGY, SPIRITUALITY, AND RELIGIOUSNESS IN MILITARY \\ CONTEXTS
}

Hugo Simkin ${ }^{1}$; Gisela Matrángolo²; Susana Azzollini ${ }^{3}$

Cómo citar este artículo / Citation: Simkin H., Matrángolo G., Azzollini S. (2018). "Ideología del rol de género, espiritualidad y religiosidad en contextos militares".

Revista Cientifica Arbitrada de la Fundación MenteClara, 3(2) abril-septiembre 2018, 75-92. DOI: https://doi.org/10.32351/rca.v3.2.55

Copyright: (c) 2018 RCAFMC. Este artículo de acceso abierto es distribuido bajo los términos de la licencia Creative Commons Attribution-Non Commercial (by-cn)

Spain 3.0. Recibido: 09/09/2018. Aceptado: 19/09/2018 Publicación online:

$19 / 10 / 2018$

Conflicto de intereses: Ninguno que declarar.

Financiación: El presente trabajo se enmarca dentro de los proyectos de Investigación de los proyectos UBACyT UBACyT20020110100222 a cargo de la Dra. Susana Azzolini y UBACyT 20020150200259BA y PICT-2016- 4147 a cargo del Dr. Hugo Simkin acreditados por la Universidad de Buenos Aires (UBA) y la Agencia Nacional de Promoción Científica y Tecnológica (ANPCyT)

\section{Resumen}

Distintos autores observan que las concepciones en relación al género en contextos militares son reforzadas por los vínculos entre las Fuerzas Armadas y las instituciones religiosas, caracterizadas tradicionalmente por la promoción de roles de género más conservadores. Sin embargo, mientras que el apego a los roles tradicionales suele asociarse positivamente con la religiosidad, su relación con la espiritualidad permanece relativamente inexplorada. Por este motivo, el presente

\footnotetext{
${ }^{1}$ Universidad de Buenos Aires. (CONICET), Argentina. e-mail: hugosimkin@psi.uba.ar

2 Universidad Maimónides, (CONICET), Argentina.

${ }^{3}$ Universidad de Buenos Aires. (CONICET), Argentina.
} 
estudio se propuso identificar si existen relaciones entre la espiritualidad, la religiosidad y la ideología del rol de género en una muestra de 102 participantes de ambos géneros con experiencia militar. Se emplearon las escalas ASPIRES e IRG para la evaluación de los constructos de interés. Los resultados sugieren que mientras que la religiosidad se encuentra asociada positivamente a creencias vinculadas a una ideología del rol de género más conservadora, la espiritualidad no se encuentra relacionada en absoluto. Las implicancias del estudio aportan al esclarecimiento del modo en que se relacionan estas variables en contextos militares y religiosos, así como a la discriminación de la espiritualidad y de la religiosidad como constructos diferentes.

\begin{abstract}
Different authors observe that the conceptions in relation to gender in military contexts are reinforced by the links between the Armed Forces and religious institutions, traditionally characterized by the promotion of more conservative gender roles. However, while higher attachment to traditional values seems to be associated with religion, it's relationship with spirituality remains unexplored. For this reason, this study aimed to explore the relationships between spirituality, religiosity and gender role ideology in a sample of 102 participants of both genders with military experience. The ASPIRES and IRG scales were used for the evaluation of the constructs of interest. The results show that while religiosity is found to be positively related to beliefs linked to a traditional gender role ideology, spirituality is not related to it at all. The findings of the study contribute to the clarification of the way in which these variables are related to each other in religious and military contexts, as well as to the discrimination of spirituality and religiosity as different constructs.
\end{abstract}

\title{
Palabras Claves/ Keywords
}

Espiritualidad; Religiosidad; ideología del rol de Genero; Contextos Militares; Spiritually; Religiosity; Ideology of de Rol Gender; Military Context 


\section{Introducción}

En sus orígenes, la explicación psicológica de las diferencias entre hombres y mujeres tenía sus raices estrictamente en aspectos de índole biológicos y naturales (Sibley \& Barlow, 2016; Ungaretti, Etchezahar, \& Simkin, 2012). Sin embargo, actualmente se considera que la influencia de la cultura a lo largo del proceso de socialización tiene un papel central en la adopción de un determinado género (Barrancos, 2014).

De acuerdo con la literatura, las diversas sociedades históricamente han categorizado a mujeres y hombres a través de estereotipos aparentemente inamovibles, promoviendo sistemas de creencias relativos a la masculinidad, a la feminidad o a las sexualidades disidentes (Carli, Alawa, Lee, Zhao, \& Kim, 2015; Gazzola \& Morrison, 2014).

De esta manera, a partir de dichos estereotipos, cada cultura establece roles de género determinados, es decir, creencias relacionadas con el papel que cada género debe desempeñar en la sociedad (Eagly \& Steffen, 1984; Kroska, 2014; Riley \& Evans, 2017).

Así, mientras que algunas ocupaciones ligadas a la agresividad, la fuerza física, o la competitividad -como las carreras políticas o militareshan sido consideradas de "naturaleza masculina" otras labores usualmente vinculadas al cuidado -como la enfermería, la educación de la primera infancia o la cosmetología- se han considerado de "naturaleza femenina" (Huffman, Culbertson, \& Barbour, 2015; McDowell, 2015; Schwartz \& Rubel-Lifschitz, 2009).

Entre las ocupaciones atribuidas más frecuentemente a los hombres se destacan aquellas que se inscriben en el ámbito las Fuerzas Armadas (Hinojosa, 2010; Kuhl, Kosloski, Ryon, \& Monar, 2018).

Distintos autores observan que las concepciones en relación al género en contextos militares son reforzadas por los vínculos entre las Fuerzas 
Armadas y las instituciones religiosas (Levy, 2010; Mallimaci, 1995), caracterizadas tradicionalmente por la promoción de roles de género más conservadores (Haggard, Kaelen, Saroglou, Klein, \& Rowatt, 2018; Nuño Martinez, 2015; Quero, 2011).

En particular, la compleja relación entre Estado y religión en Israel ha contribuido al estrecho vínculo entre las instituciones militares y religiosas desde su independencia en 1948 (Cohen, Kampinsky, \& Rosman-Stollman, 2016).

En este escenario, la considerable paridad de hombres y mujeres en las Fuerzas de Defensa de Israel motivó un amplio número de investigaciones sobre género y ejército (Feldman, 2017; Sasson-Levy, 2011).

De acuerdo con la literatura, la participación de las mujeres dentro de las Fuerzas Armadas ha generado distintas reacciones (Koch, D'Mello, \& Sackett, 2015; Nuciari, 2018; Rones \& Fasting, 2017). En las comunidades religiosas menos ortodoxas, donde se promueven roles de género menos tradicionales, la participación de las mujeres en tareas históricamente reservadas a los hombres tiene una mayor aceptación (De Los Ríos Zarzosa, 2015; Frank \& Leaman, 2005).

Por el contrario, las comunidades religiosas más ortodoxas tienden a oponerse al servicio militar para las mujeres en general, y para las religiosas en particular (De Los Ríos Zarzosa, 2015; Hartman, 2007; Kohan, 2003; Levy, 2010; Rosman-Stollman, 2018).

Por otra parte, cabe señalar que la distinción entre religiosidad y espiritualidad ha generado numerosos debates en la literatura psicológica (Oman, 2013; Zinnbauer et al., 1997). Distintos autores observan que mientras que algunas personas pueden considerarse 
religiosas y espirituales, otras pueden identificarse como espirituales, pero no religiosas (Schnell, 2012).

Si bien no existe un consenso relativo a una definición unívoca, la espiritualidad o trascendencia espiritual suele comprenderse como una motivación que orienta el comportamiento en el esfuerzo de construir un sentido más profundo para la vida desde una perspectiva escatológica, relativa al destino del ser humano luego de su muerte (Piedmont, 1999, 2004b).

Por su parte, la religiosidad suele remitir al modo en que la espiritualidad es moldeada y se expresa a través de una organización religiosa, comunitaria o social (Piedmont, 2012). Distinguir entre ambos conceptos representa uno de los mayores obstáculos en psicología de la religión para sistematizar los hallazgos reportados en las investigaciones que se ocupan de explorar sus relaciones con otros constructos psicológicos, como la ideología del rol de género (Kapuscinski \& Masters, 2010; Simkin, 2017a, 2017b).

En este escenario, el presente trabajo se propone explorar si existen relaciones entre la ideología de rol de género, la religiosidad y la espiritualidad en el marco del entrecruzamiento entre Fuerzas Armadas, Estado y religión. 


\section{Método}

\subsection{Diseño y participantes.}

La muestra fue no probabilística, incidental, compuesta por 102 participantes israelies de habla hispana con experiencia en el Servicio Militar Obligatorio con edades comprendidas entre los 19 y los 76 años $(M=37.00 ; D E=12.5)$ y de ambos sexos $($ Hombres $=25.8 \%$; Mujeres $=$ $74.8 \%$ ). Los datos fueron recolectados a través de un instrumento de evaluación de índole autoadministrable.

\subsection{Instrumentos}

Se emplearon medidas de autoinforme mediante una batería de instrumentos de evaluación que incluyó las siguientes técnicas:

Escala Abreviada de Evaluación de la Espiritualidad y los Sentimientos Religiosos / Assessment of Spirituality and Religious Sentiments Short Form (ASPIRES) (Piedmont, 2004a).

ASPIRES es un cuestionario autoadministrable de 35 ítems que evalúa dos dimensiones en población adolescente y adulta: (1) Sentimientos Religiosos (Religious Sentiments) y (2) Trascendencia Espiritual (Spiritual Transcendence). A su vez, RS se compone de dos dominios: (A) Participación Religiosa (Religiosity Scale) y (B) Crisis Religiosa (Religious Crisis), mientras que TE comprende otros tres (A) Realización en la oración (Prayer Fulfillment), (B) Universalidad (Universality) y (C) Conectividad (Connectedness).

La escala presenta un formato tipo Likert con cinco anclajes de respuesta en función del grado de acuerdo de los participantes, siendo 1 "Completamente de acuerdo" y 5 "Completamente en desacuerdo". Para el presente estudio se empleó la versión abreviada adaptada al español 
(Simkin \& Piedmont, 2018) que comprende 13 ítems extraídos de la versión original, relevando las facetas Participación Religiosa o Religiosidad, Realización en la oración, Universalidad y Conectividad (Piedmont, 2004a).

Escala de Ideología del rol de Género (EIG) (Moya, Expósito, \& Padilla, 2006).

EIG es un cuestionario autoadministrable de 12 items que evalúa la Ideología del rol de género en adolescentes y adultos. La escala presenta un formato tipo Likert con cinco anclajes de respuesta en función del grado de acuerdo de los participantes siendo 1 "Totalmente de acuerdo" y 5 “Totalmente en desacuerdo". Para el presente estudio se empleó la versión adaptada al español por Ungaretti et al. (2013).

Cuestionario de datos sociodemográficos.

Se incluyó un cuestionario ad hoc que solicita a los participantes consignar la edad, el género y el nivel de estudios

\subsection{Procedimiento}

Los sujetos fueron invitados a participar de la investigación de forma voluntaria. Se les solicitó un consentimiento informado para la participación en el estudio en el que constaba que los datos derivados de esta investigación se utilizarán exclusivamente con fines científicos.

\subsection{Análisis de datos}

Los análisis se realizaron a través del software estadístico SPSS 24. En primer lugar se realizó un análisis correlacional para explorar las relaciones entre las variables en estudio (Judd \& Kenny, 2010). 
Posteriormente, se testeó un modelo en el cual se incluyó como variable dependiente a la ideología del rol de género y como variables independientes a la religiosidad y a la trascendencia espiritual. Siguiendo las recomendaciones de la literatura (Freiberg \& Fernández, 2015), se evaluó la bondad de ajuste global del modelo tomando el $\mathrm{R}^{2}$ como un indicador del tamaño del efecto y el $\mathrm{R}^{2}$ corregido como un indicador de la varianza total explicada, ponderando asimismo la magnitud del error típico (Gil, 2011; Gutiérrez, 2007).

A continuación, se testeó si el modelo cumplía con los supuestos de no autocorrelación, multicolinealidad y homocedasticidad de los residuos empleando el test de Durbin-Watson para el examen de la no autocorrelación y los índices de condición y los factores de inflación de la varianza (FIV) para el examen de la multicolinealidad (Xin \& Gang, 2009). Finalmente, se analizaron los coeficientes de regresión del modelo para conocer el efecto de las variables independientes en la variable dependiente.

\section{Resultados}

En primer lugar, se realizó un análisis correlacional con el propósito de explorar las asociaciones entre las variables de interés (Cohen, Cohen, West, \& Aiken, 1983; Judd \& Kenny, 2010). Como se puede observar en la tabla 1, la trascendencia espiritual y la religiosidad se encuentran positivamente asociadas entre sí. Sin embargo, mientras que la ideología del rol de género se encuentra vinculada a la religiosidad, no presenta relaciones con la espiritualidad que sean estadísticamente significativas. 


\section{Tabla 1}

Correlaciones entre la espiritualidad, la religiosidad y la ideología del rol de género

$1 \quad 2 \quad 3$

1. Religiosidad

2. Trascendencia Espiritual

3.Ideología del Rol de Genero
$.27^{*}$

$.36^{*} \quad .21$

${ }^{* *} \cdot p<.01 ; * . p<.05$

En segundo lugar, se testeó un modelo en el cual se incluyó como variable dependiente a la ideología del rol de género y como variables independientes a la religiosidad y a la trascendencia espiritual.

Se aplicó el método de pasos hacia adelante a partir del cual se obtuvo un modelo que incluyó solamente una explicación significativa de la variable religiosidad sobre ideología del rol de género, quedando excluida la espiritualidad (Berlanga-Silvente \& Vilà-Baños, 2014; Coolican, 2014). Tal como ha sido sugerido en la literatura (Freiberg \& Fernández, 2015), se evaluó la bondad de ajuste global del modelo tomando el $\mathrm{R}^{2}$ como un indicador del tamaño del efecto y el $\mathrm{R}^{2}$ corregido como un indicador de la varianza total explicada (Darlington \& Hayes, 2016; Gil, 2011).

Asimismo, se ponderó la magnitud del error típico (Gutiérrez, 2007; Schroeder, Sjoquist, \& Stephan, 2016). 


\section{Tabla 2}

Ajuste global del modelo predictivo de la ideología del rol de género

$\mathrm{R}$ .362

$\mathrm{R}^{2}$

$\mathrm{R}^{2}$ corregido

Error típico

Durbin-Watson

1.84

A continuación, se testeó si el modelo cumplía con los supuestos de multicolinealidad, homocedasticidad de los residuos y no autocorrelación con el objeto de confirmar el ajuste del modelo estimado (Freiberg \& Fernández, 2015; Xin \& Gang, 2009).

De acuerdo con los antecedentes se empleó el test de Durbin-Watson para el examen de la no autocorrelación. Asimismo, se emplearon los índices de condición y los factores de inflación de la varianza (FIV) para el examen de la multicolinealidad. Tal como se puede observar en las tablas 2 y 3 , el cumplimiento de los supuestos permite constatar el ajuste correcto del modelo.

\section{Tabla 3}

Índices de multicolinealidad

\section{Índices Condición $\quad F I V$}

Religiosidad

3.731

1.000 
Respecto del efecto de las variables independientes en la variable dependiente, tal como se observa en la tabla 4, el estadístico B permite informar acerca de la medida en la que varía la ideología del rol de género respecto de la religiosidad y de la espiritualidad.

El coeficiente Beta fue empleado como estimador de la magnitud en la que las variables independientes resultaron predictoras de la variabilidad en la religiosidad como variable dependiente.

La prueba t, así como su nivel de significación, permitió conocer si el efecto de la variable explicativa -religiosidad- hacia la variable explicada -ideología del rol de género- resultaba estadísticamente significativo. De acuerdo con los resultados reportados en la tabla 4 se puede observar que la religiosidad se presenta como la variable explicativa que integra el modelo.

\section{Tabla 4}

Coeficientes de regresión del modelo

\begin{tabular}{|c|c|c|c|c|c|c|}
\hline & $B$ & IC $95 \%$ & $\begin{array}{l}\text { Error } \\
\text { Tipico }\end{array}$ & Beta & $t$ & Sig \\
\hline (constante) & 5.137 & $\begin{array}{l}{[3.548,} \\
6.727]\end{array}$ & .795 & & 6.465 & .000 \\
\hline Religiosidad & 1.158 & $\begin{array}{c}{[.389} \\
1.927]\end{array}$ & .384 & .362 & 3.012 & .004 \\
\hline
\end{tabular}




\section{Discusión}

Tal como se ha sugerido en la literatura, la espiritualidad y la religiosidad se presentan como constructos relacionados, pero diferentes. El vínculo entre una ideología de género más conservadora y una mayor religiosidad aportaría apoyo empírico a los estudios que sugieren que las comunidades ortodoxas continúan trasmitiendo un conjunto de valores tradicionales en relación a los roles de los hombres y las mujeres en la sociedad.

Cabe destacar que no se observan relaciones entre la espiritualidad y la ideología de género, lo que contribuye a sostener la especificidad de cada uno de los constructos numinosos.

De esta manera, mientras que numerosos individuos pueden identificarse como espirituales y religiosos, otros pueden percibirse como espirituales, pero no religiosos.

La implicancia de estas diferencias resulta de enorme relevancia para la psicología de la religión, en tanto parte de la dificultad de sistematizar los resultados de las investigaciones en el área deriva de los obstáculos reportados para arribar a categorias consensuadas que permitan relevar los fenómenos vinculados a las experiencias numinosas.

No sólo es fundamental comprender a la religiosidad y la espiritualidad como constructos vinculados pero independientes, sino que, en el presente trabajo se considera necesario, además, atender al complejo vínculo entre Estado y religión que se expresa en gran medida en las tensiones relativas al rol de las mujeres en las Fuerzas Armadas.

Estas tensiones resultan aún más complejas en el marco de los debates internos del feminismo entre quienes reivindican (Lucero, 2009) y quienes rechazan la participación de las mujeres en las instituciones militares (Arribas, 1999; De Sevilla, 2009). 
Entre las limitaciones del estudio se destaca que, si bien las técnicas empleadas permiten la distinción entre la religiosidad y la espiritualidad (Piedmont, 2004a) no alcanzan a relevar la enorme diversidad de los fenómenos religiosos que describe la literatura especializada en relación, por ejemplo, a las diferentes posturas sobre el tema adoptadas por comunidades religiosas de distintos niveles de ortodoxia (De Los Ríos Zarzosa, 2015; Frank \& Leaman, 2005; Hartman, 2007; Kohan, 2003). En este sentido, futuros estudios podrian ocuparse de incluir otras escalas que permitan abordar la diversidad de las experiencias religiosas con mayor detalle (Batson \& Schoenrade, 1991a, 1991b). 


\section{Referencias}

Arribas, J. L. C. de M. (1999). Antimilitarismo y feminismo: Las mujeres, La campaña de insumisión y 25 años desobedeciendo. In A. Aguado (Ed.), Mujeres, Regulación de conflictos sociales y cultura de la paz, (1999) (Universita, pp. 177-200). Valencia.

Barrancos, D. (2014). Géneros y sexualidades disidentes en la Argentina: de la agencia por los derechos a la legislación positiva 1. Cuadernos Inter.c.a.Mbio Sobre Centroamérica y El Caribe, 11(2), 17-46.

Batson, C. D., \& Schoenrade, P. (1991a). Measuring Religion as Quest-Reliability Concerns. Journal for the Scientific Study of Religion.

Batson, C. D., \& Schoenrade, P. (1991b). Measuring Religion as Quest-Validity Concerns. Journal for the Scientific Study of Religion.

Berlanga-Silvente, V., \& Vilà-Baños, R. (2014). Cómo obtener un Modelo de Regresión Logística Binaria con SPSS. REIRE. Revista d'Innovació i Recerca En Educació, 7(2), 105118. https://doi.org/10.1344/reire2014.7.2727//-

Carli, L. L., Alawa, L., Lee, Y. A., Zhao, B., \& Kim, E. (2015). Stereotypes About Gender and Science: Women $\neq$ Scientists. Psychology of Women Quarterly, 4O(2), 244-260. https://doi.org/10.1177/0361684315622645

Cohen, J., Cohen, P., West, S. G., \& Aiken, L. S. (1983). Applied multiple regression/correlation analysis for the behavioral sciences. New York: Psychology Press.

Cohen, S., Kampinsky, A., \& Rosman-Stollman, E. (2016). Swimming against the tide: the changing functions and status of chaplains in the Israel Defense Force. Religion, State and Society, 44(1), 65-74. https://doi.org/10.1080/09637494.2015.1122881

Coolican, H. (2014). Research methods and statistics in psychology. London: Psychology Press.

Darlington, R. B., \& Hayes, A. F. (2016). Regression analysis and linear models: Concepts, applications, and implementation. New York: The Guilford Press.

De Los Ríos Zarzosa, G. S. (2015). Judaísmo y judaísmos: Una realidad plural y compleja. Ilu, 20, 249-268. https://doi.org/10.5209/rev-ILUR.2015.v20.50412

De Sevilla, M. de N. (2009). Edita: Coordinadora Estatal de Organizaciones Feministas. In Granada, treinta años despues. Aqui y ahora. Jornadas Feministas Estatales. (pp. 105112). 
Eagly, A. H., \& Steffen, V. J. (1984). Gender stereotypes stem from the distribution of women and men into social roles. Journal of Personality and Social Psychology, 46(4), 735-754. https://doi.org/10.1037/0022-3514.46.4.735

Feldman, J. (2017). "Point Men": How religious women soldiers may restore Israel's "Citizens' Army". The Journal of the Middle East and Africa, 8(4), 325-351. https://doi.org/10.1080/21520844.2017.1401872

Frank, D. H., \& Leaman, O. (2005). History of Jewish Philosophy. Routledge : Londres

Freiberg, A., \& Fernández, M. M. (2015). Estilos de aprendizaje en estudiantes universitarios ingresantes y avanzados de Buenos Aires. Liberabit, 21(1), 71-79.

Gazzola, S. B., \& Morrison, M. A. (2014). Cultural and Personally Endorsed Stereotypes of Transgender Men and Transgender Women: Notable Correspondence or Disjunction? International Journal of Transgenderism, 15(2), 76-99. https://doi.org/10.1080/15532739.2014.937041

Gil, J. A. (2011). Metodología cuantitativa en educación. Madrid: Universidad Nacional de Educación a Distancia.

Gutiérrez, J. C. A. (2007). Estadistica general aplicada. Medellin: Escuela de Administración, Finanzas e Instituto Tecnológico.

Haggard, M. C., Kaelen, R., Saroglou, V., Klein, O., \& Rowatt, W. C. (2018). Religion's role in the illusion of gender equality: Supraliminal and subliminal religious priming increases benevolent sexism. Psychology of Religion and Spirituality. https://doi.org/10.1037/re10000196

Hartman, T. (2007). Feminism Encounters Traditional Judaism: Resistance and Accommodation. University Press New England: Londres.

Hinojosa, R. (2010). Doing Hegemony: Military, Men, and Constructing a Hegemonic Masculinity. The Journal of Men's Studies, 18(2), 179-194. https://doi.org/10.3149/jms. 1802.179

Huffman, A. H., Culbertson, S. S., \& Barbour, J. (2015). Gender Roles in a Masculine Occupation: Military Men and Women's Differential Negotiation of the Work-Family Interface. In Gender and the Work-Family Experience (pp. 271-289). Cham: Springer International Publishing. https://doi.org/10.1007/978-3-319-08891-4_14

Judd, C. M., \& Kenny, D. A. (2010). Data analysis in social psychology: Recent and recurring issues. In S. T. Fiske, D. T. Gilbert, \& L. Gardner (Eds.), Handbook of Social Psychology (pp. 115-139). New Jersey: Wiley. 
Kapuscinski, A. N., \& Masters, K. S. (2010). The current status of measures of spirituality: A critical review of scale development. Psychology of Religion and Spirituality, 2(4), 191205. https://doi.org/10.1037/a0020498

Koch, A. J., D’Mello, S. D., \& Sackett, P. R. (2015). A meta-analysis of gender stereotypes and bias in experimental simulations of employment decision making. Journal of Applied Psychology, 100(1), 128-161. https://doi.org/10.1037/a0036734

Kohan, G. (2003). La mujer y el trabajo en el Judaismo. THÉMATA, Revista de Filosofia, 1 (31), 89-99. Universidad de Sevilla

Kroska, A. (2014). The Social Psychology of Gender Inequality. In J. D. McLeod, E. J. Lawler, \& M. Schwalbe (Eds.), Handbook of the social psychology of inequality (pp. 485515). New York: Springer.

Kuhl, S., Kosloski, A., Ryon, S., \& Monar, A. (2018). Masculinity, Organizational Culture, Media Framing and Sexual Violence in the Military. Social Sciences, 7(5), 80. https://doi.org/10.3390/socsci7050080

Levy, Y. (2010). The Clash between Feminism and Religion in the Israeli Military: A Multilayered Analysis. Social Politics: International Studies in Gender, State \& Society, 17(2), 185-209. https://doi.org/10.1093/sp/jxq002

Lucero, M. R. (2009). Las Mujeres En Las Fuerzas Armadas Argentinas. Breve Análisis Sobre Su Participación Y Estado Actual De La Situación. UNISCI Discussion Papers, (20), 36-49.

Mallimaci, F. (1995). Catolicismo y militarismo en la Argentina 1930-1983. Revista de Ciencias Sociales, 4, 1-23.

McDowell, J. (2015). Masculinity and Non-Traditional Occupations: Men's Talk in Women's Work. Gender, Work \& Organization, 22(3), 273-291.

https://doi.org/10.1111/gwao.12078

Moya, M., Expósito, F., \& Padilla, J. L. (2006). Revisión de las propiedades psicométricas de las versiones larga y reducida de la Escala sobre Ideología de Género. International Journal of Clinical and Health Psychology, 6(3), 709-727.

Nuciari, M. (2018). Participation and Change in Gendered Organisations. Women in the Military Forces. In G. Caforio \& M. Nuciari (Eds.), Handbook of the Sociology of the Military (pp. 301-325). Cham: Springer. https://doi.org/10.1007/978-3-319-716022_15 
Nuño Martinez, N. (2015). La cuestión de género en la religión Hare Krishna : una aproximación a su construcción teológica y antropológica, sus conflictos y debates actuales. Revista Clepsydra, 14, 95-114.

Oman, D. (2013). Defining Religion and Spirituality. In R. F. Paloutzian \& C. L. Park (Eds.), Handbook of the Psychology of Religion and Spirituality (pp. 23-48). New York: The Guilford Press.

Piedmont, R. L. (1999). Does Spirituality Represent the Sixth Factor of Personality? Spiritual Transcendence and the Five-Factor Model. Journal of Personality, 67(6), 9851013. https://doi.org/10.1111/1467-6494.00080

Piedmont, R. L. (2004a). Assessment of spirituality and religious sentiments, technical manual (1st ed.). Timonium, Maryland: Author.

Piedmont, R. L. (2004b). The Logoplex as a paradigm for understanding spiritual transcendence. Research in the Social Scientific Study of Religion, 15(1), 263-284. https://doi.org/10.1037/0893-164X.18.3.213

Piedmont, R. L. (2012). Overview and Development of Measure of Numinous Constructs: The Assessment of Spirituality and Religious Sentiments (ASPIRES) Scale. In L. J. Miller (Ed.), The Oxford Handbook of Psychology and Spirituality (pp. 104-122). Oxford: Oxford University Press.

Quero, H. C. (2011). Sexualizando la Trinidad: Aportes desde una teología de la liberación queer a la compresión del misterio divino. Cuadernos de Teología, XXX, 53-70.

Riley, S., \& Evans, A. (2017). Gender. In B. Gough (Ed.), The Palgrave Handbook of Critical Social Psychology (pp. 409-433). London: Palgrave McMillan.

Rones, N., \& Fasting, K. (2017). Theorizing Military Masculinities and National Identities: The Norwegian Experience. In The Palgrave International Handbook of Gender and the Military (pp. 145-162). London: Palgrave Macmillan UK. https://doi.org/10.1057/978-1137-51677-0_9

Rosman-Stollman, E. (2018). Military Service as Bargaining: The Case of Religious Women Soldiers in Israel. Politics, Religion \& Ideology, 19(2), 158-175. https://doi.org/10.1080/21567689.2018.1448797

Sasson-Levy, O. (2011). Research on Gender and the Military in Israel: From a Gendered Organization to Inequality Regimes. Israel Studies Review, 26(2). https://doi.org/10.3167/isr.2011.260205

Schnell, T. (2012). Spirituality with and without Religion-Differential Relationships with Personality. Archive for the Psychology of Religion, 34(1), 33-61. https://doi.org/10.1163/157361212X644495 
Schroeder, L. D., Sjoquist, D. L., \& Stephan, P. E. (2016). Understanding Regression Analysis. Los Angeles.

Schwartz, S. H., \& Rubel-Lifschitz, T. (2009). Cross-national variation in the size of sex differences in values: Effects of gender equality. Journal of Personality and Social Psychology, 97(1), 171-185. https://doi.org/10.1037/a0015546

Sibley, C. G., \& Barlow, F. K. (2016). An Introduction to the Psychology of Prejudice. In C. G. Sibley \& F. K. Barlow (Eds.), The Cambridge Handbook of the Psychology of Prejudice (pp. 3-20). Cambridge: Cambridge University Press. https://doi.org/10.1017/9781316161579.001

Simkin, H. (2017a). La salud mental en la psicología de la religión y de la espiritualidad. Revista Científica Arbitrada de La Fundación MenteClara, 2(2), 169-204.

Simkin, H. (2017b). Psicología de la Religión: adaptación y validación de escalas de evaluación psicológica en el contexto argentino. Anuario de Investigaciones, 24, 177-185.

Simkin, H., \& Piedmont, R. L. (2018). Adaptation and Validation of the Assessment of Spirituality and Religious Sentiments (ASPIRES) scale short form into spanish. Latinoamerican Journal of Positive Psychology, 4, 97-107.

Ungaretti, J., Etchezahar, E., \& Simkin, H. (2012). El estudio del prejuicio desde una perspectiva psicológica: cuatro períodos histórico-conceptuales para la comprensión del fenómeno. Calidad de Vida, 8(1), 3-30.

Ungaretti, J., Etchezahar, E., \& Simkin, H. (2013). El prejuicio sexista hacia la mujer desde la ideología del rol de género. In B. Kerman \& G. Michelini (Eds.), El impacto de la investigación en la Universidad sobre la Sustentabilidad Social y Ambiental (1st ed., pp. 69-75). Buenos Aires: Universidad de Flores.

Xin, Y., \& Gang, X. (2009). Linear regression analysis. Singapur: World Scientific.

Zinnbauer, B. J., Pargament, K. I., Cole, B., Rye, M. S., Eric, M., Belavich, T. G., Butter, E. M. (1997). Religion and Spirituality: Unfuzzying the Fuzzy. Journal for the Scientific Study of Religion, 36(4), 549-564. https://doi.org/10.2307/ 1387689 